given OME's asympotomatic presentation, requires long-term ENT and audiological monitoring at least until the age of 3 years.

\section{LUNG SEQUESTRATION IN PATIENT WITH TRANSPOSITION OF THE GREAT ARTERIES (D-TGA) - WHY IS IMPORTANT TO DIAGNOSE THIS LUNG ANOMALY}

Sanja Dorner*, Ivan Pavić, Borna Biljan, Igor Nikolić. University Hospital Osijek, Pediatric Clinic, Medical Faculty Osijek

\subsection{6/archdischild-2021-europaediatrics.35}

In this case we present a patient who was hospitalized in our hospital at the age of 3 hours due to progressive respiratory failure and cyanosis. The birth occurred spontaneously at 35 weeks of gestation, BM $3020 \mathrm{~g}$, BL $50 \mathrm{~cm}$, Apgar 3,7. Immediately after birth neonate was tachidispnoic and generally cyanotic. Despite application of surfactant and mechanical ventilation, oxygen saturation does not improve. Echocardiography revealed transposition of the great arteries (d-TGA). After a balloon atrioseptostomy, a short-term improvement occurs, but soon the right-sided pneumothorax develops and after its resolution patient was sent to a foreign cardiac surgery center where a complete cardiac surgery correction of TGA was successfully made. The postoperative course was complicated by sinus thrombosis and echocardiography shows an excellent postoperative finding.

The patient continues treatment at a local hospital which was closer to his residence and where was retreated almost every 2 months for the next 4 years because of recurrent bronchoconstriction and right-sided pneumonia.

Extensive allergologically immune diagnostic treatment has been made due to suspected immunodeficiency and allergic lung disease. During such an episode of recurrent right-sided pneumonia, as there was no improvement in treatment, patient was transferred to our facility. Immediately upon arrival, a CT scan of the chest was performed to detect the existence of an intralobar pulmonary sequester in the upper and posterobasal segment of the lower lung lobe. A thoracotomy was performed and the lung sequester was surgically removed, without complications. Since then, our patient is healthy, has not had a single respiratory infection in the last 2 years, which has significantly improved his quality of life. Lung sequestration is a rare congenital lung anomaly that belongs to the group of congenital cystic malformations with an incidence of $0.15 \%-6.4 \%$. There is two forms of sequestration - intralobar and extralobar. Symptoms of the disease are often atypical and disease is often unrecognized, according to literature data, in $71 \%$ of cases, which significantly affects on success of treatment and the quality of life of these patients. Therefore, it is necessary for physicians, especially pediatricians, to raise awareness of this disorder so it can be diagnosed and adequately cured. Some authors even recommend surgical treatment before the onset of the problem as soon as it is diagnosed, to avoid the possible complications, such as hemothorax, fungal and TB infection, malignant tissue alteration in the sequester.

\section{SERUM CALPROTECTIN IS A NOVEL PREDICTOR OF BACTERIAL URINARY TRACT INFECTION IN A FEBRILE CHILD}

Mirta Lamot*, Marijana Miler, Nora Nikolac Gabaj, Ivana Trutin, Renata Vrsalović, Iva Mihatov Štefanović, Biserka Čičak, Inga Sitaš, Mandica Vidović, Lovro Lamot, Slaven Abdović, Miroslav Harjaček. Sestre milosrdnice University Hospital Center

\subsection{6/archdischild-2021-europaediatrics.36}

Although self-limiting viral infections are the principal cause of fever in the majority of cases, it is estimated that $5 \%$ of febrile children younger than three years of age would have a urinary tract infection (UTI). Despite the increased availability of laboratory testing and development of various clinical scores, there is still no reliable predictor of UTI in children.

Recent advancements in the understanding of inflammatory process have highlighted the role of S100 proteins, with many studies suggesting their clinical value as a biomarker of inflammation. This research aims to investigate the possible role of serum calprotectin (sCAL), a heterodimer consisting of S100A8 and S100A9 proteins from S100 family, as a biomarker for bacterial urinary tract infection in children.

Patients aged 0-36 months who came to Pediatric Emergency Department of Sestre milosrdnice University Hospital Center with fever $\geq 38^{\circ} \mathrm{C}$, lasting $<72 \mathrm{~h}$, positive urine leukocyte esterase and no history of chronic illness or ongoing antibiotic use, were enrolled. In every patient, sCAL was measured with Quantum Blu rapid test, along with urine culture analysis and testing of standard inflammatory markers. Patients with bacterial count of $\geq 10^{\wedge} 5 \mathrm{CFU} / \mathrm{mL}$ in urine culture were defined as having UTI, while patients with negative culture, absent signs of other bacterial infection, positive influenza, adenovirus, and/or respiratory syncytial virus test, and/or characteristic symptoms were diagnosed with viral acute respiratory infection (ARI).

Among 52 patients included in the study (median age 6 months, IQR 3,1 - 13,8), 30 were diagnosed with ARI and 22 with UTI. In patients with UTI, the median value of sCAL was 5,6 ug/mL (IQR 3,9 - 6,3), which was significantly higher $(p<0,0001)$ than in patients with ARI, whose median value of sCAL was 2,6ug/mL (IQR 1,7 - 3,5). There was a moderate positive correlation of $\mathrm{sCAL}$ with WBC $(\mathrm{r}=0,64 ; \mathrm{p}<0,01)$ and CRP $(\mathrm{r}=0,55 ; \mathrm{p}<0,01)$. The sCAL had area under curve (AUC) of 0.93 (95\% CI 0.85 - 100) for predicting UTI, and with the optimal cut-off $(3,95 \mathrm{ug} / \mathrm{mL})$, sensitivity was $93,1 \%$ (95\% CI

78,0 - 98,8\%), specificity 76,19\% (95\% CI 54,9 - 89,4\%) and positive likelihood ratio was 3,91 .

The results of this study have shown that sCAL is a good predictor of bacterial UTI in a febrile child presenting to an emergency department.

Besides, the correlation of sCAL with duration of fever and inflammatory markers standardly used to infer the presence of bacterial infection was only moderate or insignificant, suggesting the potential differences in their dynamics. Hence, the sCAL concentration in febrile children could add to timely and accurate diagnosis of UTI, which is essential to reduce treatment delay and improve treatment outcomes. 